Pacific Journal of Mathematics

THE SCHUR GROUP OF A FIELD OF CHARACTERISTIC
ZERO 


\section{THE SCHUR GROUP OF A FIELD OF CHARACTERISTIC ZERO}

\section{R. MOLLIN}

We determine when a class in the Schur subgroup $S(K)$ of the Brauer group $B(K)$ of a field $K$ of characteristic zero contains an algebra which is isomorphic to a simple summand $A$ of the group algebra $F G$ for some finite group $G$, where $F$ is a subfield of $K$. We then investigate $A \otimes_{F} K$ which is the direct sum of simple algebras with center $K$, and determine exactly when these are $K$-isomorphic. Finally we refine existing examples in the theory of the Schur group, and obtain a decomposition theorem for the related group of algebras with uniformly distributed invariants.

In the introduction to [6] Janusz notes that: For a finite abelian extension $K$ of the rationals $Q, S(K)$ the Schur subgroup of the Brauer group $B(K)$ consists of all classes $[A]$ consisting of an algebra $A$ which is isomorphic to a simple summand of the group algebra $Q G$ for some finite group $G$. Our first result in this paper is that for an arbitrary field $K$ of characteristic zero the above is false.

In Mollin [8-13] we develop the concept of the uniform distribution group $U(K)$ for an algebraic number field $K$. If $\varepsilon_{p^{a}}$ denotes a primitive $p^{a}$ th root of unity and $\varepsilon_{p^{a}}$ is the highest $p$-power root of unity in $K$ we have from Mollin [8] that when $p$ does not divide $\left|K: Q\left(\varepsilon_{p a}\right)\right|$ then:

$$
U(K)_{p}=S(K)_{p}=K \otimes S\left(Q\left(\varepsilon_{p^{a}}\right)\right)_{p}
$$

where $G_{p}$ denotes the $p$-primary part of a group $G$. In Janusz [7] it is shown that if $p$ does not divide $\left|Q\left(\varepsilon_{n}\right): K\right|$ when $Q\left(\varepsilon_{n}\right)$ is the smallest root of unity field containing $K$ then:

$$
S(K)_{p}=K \otimes S\left(Q\left(\varepsilon_{p^{a}}\right)\right)_{p} .
$$

C. Ford and G. Janusz [5] give for each prime $p$, examples of fields $K$ for which $(* *)$ does not hold. In this paper we present, for each prime $p$, fields $K$ for which the second equality of (*) does not hold but for which the first equality does hold. Finally, we obtain a decomposition theorem for $U(K)$.

2. Notation and preliminaries. Let $K$ be a field of characteristic zero. The Schur group $S(K)$ may be described as consisting of those equivalence classes in $B(K)$ which contain a simple component of the group algebra $K G$ for some finite group $G$. By 
Yamada [16, Cor. 3.11, p. 33] this is equivalent to $S(K)$ being the subgroup of $B(K)$ consisting of those equivalence classes which contain a cyclotomic algebra; i.e., a crossed product of the form $(K(\varepsilon) / K, \alpha)$ where $\varepsilon$ is a root of unity and $\alpha$ is a factor set whose values are roots of unity in $K(\varepsilon)$.

In general we denote a crossed product by $(L / K, \beta)$ which is the central simple $K$-algebra having $L$-basis $u_{\tau}, \tau \in G(L / K)$, the Galois group of $L / K$, subject to:

$$
u_{\tau} u_{\gamma}=\beta(\tau, \gamma) u_{\tau \gamma}, \quad u_{\tau} x=\tau(x) u_{\tau}
$$

for $x \in L$.

For further information on crossed products the reader is referred to Reiner's book [14].

Now, if $G$ is a finite group and $X$ is an irreducible character of $G$ we shall denote the simple component of $K G$ corresponding to $X$ as $A(X, K)$. By Yamada [16, Prop. 1.1, p. 4], $A(X, K)=K G a(X)$ with $a(X)=\sum e\left(X^{\tau}\right)$ where the sum ranges over $\tau \in G(K(X) / K)$. $a(X)$ is a primitive central idempotent of $K G$, and $K(X)$ is the center of $A(X, K)$.

$$
e(X)=X(1)|G|^{-1} \sum_{g \in G} X\left(g^{-1}\right) g
$$

3. The Schur group. We let $K$ be a field of characteristic zero throughout this section.

DEFINITION 3.1. Let $K / L$ be cyclotomic, i.e., there is a root of unity $\varepsilon$ such that $L \subseteq K \cong L(\varepsilon)$. The Schur subgroup $S_{L}(K)$ of $K$ relative to $L$ is the subgroup of $S(K)$ consisting of those classes that contain an algebra which is isomorphic to a simple summand of $L G$ for some finite group $G$.

We note here that, in fact, $S_{L}(K)$ is nonempty if and only if $K / L$ is cyclotomic. Now we ask whether or not $S_{L}(K)$ is a proper subgroup of $S(K)$. The answer is the content of the next theorem.

THEOREM 3.2. If $K / L$ is cyclotomic then $S(K)=S_{L}(K)$.

Proof. Let $[A] \in S(K)$. By Yamada [16, Cor. 3.11, p. 33] we may assume that $A=(K(\varepsilon) / K, \beta)$. But, by hypothesis $K \leqq L\left(\varepsilon^{\prime}\right)$ for some root of unity $\varepsilon^{\prime}$. Thus, using the inflation map of cohomology theory we get $[A]=[B]$ where

$$
B=\left(L\left(\varepsilon \varepsilon^{\prime}\right) / K, \beta\right)=\sum L\left(\varepsilon \varepsilon^{\prime}\right) u_{\sigma}
$$

where the direct sum ranges over all $\tau \in \mathscr{G}=G\left(L\left(\varepsilon \varepsilon^{\prime}\right) / K\right)$. 
Now, the values of $\beta$ and $\varepsilon \varepsilon^{\prime}$ generate a cyclic group $\left\langle\varepsilon^{\prime \prime}\right\rangle$ in $L^{*}\left(\varepsilon \varepsilon^{\prime}\right)$, the multiplicative group of $L\left(\varepsilon \varepsilon^{\prime}\right)$. Moreover, $\mathscr{G}$ can be regarded as an automorphism group of $\left\langle\varepsilon^{\prime \prime}\right\rangle$ where the values of $\beta$ belong to $\left\langle\varepsilon^{\prime \prime}\right\rangle$. Therefore, by the theory of group extensions (e.g., Zassenhaus [17, III, §6]) we have the exact sequence

$$
1 \longrightarrow\left\langle\varepsilon^{\prime \prime}\right\rangle \longrightarrow G \longrightarrow \mathscr{G} \longrightarrow 1
$$

where $G$ is the multiplicative subgroup of $B^{*}$ generated by $\varepsilon^{\prime \prime}$ and the elements $u_{\sigma}(\sigma \in \mathscr{G})$. In other words, $G$ is an extension of $\left\langle\varepsilon^{\prime \prime}\right\rangle$ by $\mathscr{G}$. Since $G$ spans $B$ with coefficients in $L$ then there is an $L$ algebra homomorphism of $L G$ onto $B$. Hence $B$ is isomorphic to a simple summand of $L G$.

The following is immediate.

CoROLLARY 3.3. If $K / L$ is cyclotomic and $L \subseteq T \subseteq K$ then $S(K)=S_{T}(K)$.

TheOREM 3.4. If $K / L$ is cyclotomic then

$$
S_{L}(K)=\bigcup_{L_{0}} S_{Q}\left(L_{0}\right) \bigotimes_{L_{0}} K
$$

where $L_{0}$ ranges through cyclotomic extensions of $Q$ such that $L L_{0}=K$.

Proof. It suffices to prove $S_{L}(K) \subseteq \bigcup_{L_{0}} S_{Q}\left(L_{0}\right) \otimes_{L_{0}} K$. Let $[A] \epsilon$ $S_{L}(K)$ with $A=A(X, L)$, say. Thus $K \cong L(X)$, by Dornhoff [3, Lemma 24.7, p. 124]. Now:

$$
Q G=A\left(X_{0}, Q\right) \oplus A\left(X_{1}, Q\right) \oplus \cdots \oplus A\left(X_{n}, Q\right) .
$$

For simplicity we set $A\left(X_{i}, Q\right)=A_{i}, i=0,1,2, \cdots, n ; X_{0}=X$, $Q(X)=L_{\mathrm{r}}$ and $A(X, Q)=A_{0}$. Thus:

$$
L G \cong Q G \bigotimes_{Q} L \cong A_{0} \bigotimes_{Q} L \oplus \cdots \oplus A_{n} \otimes_{Q} L
$$

and

$$
\begin{aligned}
A_{0} \bigotimes_{Q} L & \cong\left(A_{0} \bigotimes_{L_{0}} L_{0}\right) \bigotimes_{Q} L \\
& \cong A_{0} \bigotimes_{L_{0}}\left(L_{0} \bigotimes_{Q} L\right) \\
& \cong A_{0} \bigotimes_{L_{0}} L(X) \oplus \cdots \oplus A_{0} \bigotimes_{L_{0}} L(X) \\
& \cong A_{0} \bigotimes_{L_{0}} K \oplus \cdots \oplus A_{0} \bigotimes_{L_{0}} K
\end{aligned}
$$

where the latter summands correspond to $X^{\sigma}, \sigma \in G((Q(X) \cap L) / Q)$. We note that by Yamada [16, Prop. 1.5, p. 8] we have $A_{0} \cong A\left(X, L_{0}\right)$. Thus $\left[A_{0}\right] \in S_{Q}\left(L_{0}\right)$. We have shown that: 


$$
A=A(X, L) \cong A_{0} \otimes_{L_{0}} K \text { as required . }
$$

As mentioned in the introduction we do not have in general that $S_{Q}(K)=S(K)$. The following example illustrates this assertion, and we thereafter determine exactly when $S_{Q}(K)=S(K)$.

If $K=Q\left(\sqrt[3]{2}, \varepsilon_{3}\right)$ where $\sqrt[3]{2}$ is a real root of $f(x)=x^{3}-2$, $L=Q(\sqrt[3]{2})$ and $[A] \in S(K)$, then by Theorem 3.2 we have $[A] \in S_{L}(K)$. If $K$ were cyclotomic over $Q$ then $K \subseteq Q(\varepsilon)$ for some root of unity $\varepsilon$. However, this means that $K / Q$ is abelian, which is a contradiction. In fact, by the Kronecker-Weber theorem (e.g., see Ribenboim $[15$, p. 233]) an algebraic number field $K$ is cyclotomic over $Q$ if and only if $K / Q$ is abelian. In this case we could use the KroneckerWeber theorem and the method of proof of Theorem 3.2 to show that $S_{Q}(K)=S(K)$.

Now we let $[A] \in S_{L}(K)$ and $G(K / L)=\left\{\sigma_{1}=1, \sigma_{2}, \cdots, \sigma_{n}\right\}$, then $A(X, L) \otimes_{L} K \cong A\left(X^{\sigma_{1}}, K\right) \oplus \cdots \oplus A\left(X^{\sigma_{n}}, K\right)$. Since $A\left(X^{\sigma_{i}}, K\right)=$ $K G e\left(X^{\sigma_{i}}\right), e\left(X^{\sigma_{i}}\right)=X(1)|G|^{-1} \sum X^{\sigma_{i}}\left(g^{-1}\right) g$ then $A(X, K)$ is $L$-isomorphic to $A\left(X^{\sigma_{i}}, K\right)$ for $i=1,2, \cdots, n$. We ask: Are these algebras $K$-isomorphic? To illustrate that in general the answer is negative we give the following example.

We let $K=Q\left(\varepsilon_{3}\right)$ then $L=Q$ and $G(K / Q)=\langle\sigma\rangle$ where $\sigma\left(\varepsilon_{3}\right)=$ $\varepsilon_{3}^{-1}$. If the index of $A(X, K)$ is 3 then $[A(X, K)] \neq\left[A\left(X^{\sigma}, K\right)\right]$ in $B(K)$. Hence $A(X, K)$ and $A\left(X^{o}, K\right)$ are not $K$-isomorphic.

The following tells us exactly when $A\left(X^{\sigma}, K\right)$ and $A=A(X, K)$ are $K$-isomorphic.

TheOREM 3.5. Let $[A(X, K)] \in S_{L}(K)$ and let $\sigma \in G(K / L)$ then $[A(X, K)]=\left[A\left(X^{\sigma}, K\right)\right]$ if and only if $\sigma$ fixes $\varepsilon_{m}$ where $m$ is the exponent of $A$.

Proof. By Yamada $[16$, p. 14] we may assume $[A]=[A(\beta)]=$ $[(K(\varepsilon) / K, \beta)]$ where $(K(\varepsilon) / K, \beta)=\sum K(\varepsilon) u_{\tau}$ with the direct sum ranging over all $\tau \in G(K(\varepsilon) / K)$.

Now, $\sigma$ can be extended to $G(K(\varepsilon) / L)$ and we maintain the notation $\sigma$ for this automorphism.

Put:

$$
\beta^{\sigma}(\tau, \gamma)=(\beta(\tau, \gamma))^{\sigma} \text { for } \tau, \gamma \in G(K(\varepsilon) / K),
$$

then $\beta^{\sigma}$ is a factor set of $K(\varepsilon) / K$ because $G(K(\varepsilon) / K)$ is central in $G(K(\varepsilon) / L)$.

We let:

$$
A\left(\beta^{\sigma}\right)=\left(K(\varepsilon) / K, \beta^{\sigma}\right)=\sum K(\varepsilon) v_{0}
$$

where $v_{\gamma} v_{\tau}=\beta^{o}(\gamma, \tau) v_{\tau \tau} ; \gamma, \tau \in G(K(\varepsilon) / K)$. 
Now, if the values of $\beta$ generate a group $\left\langle\varepsilon_{n}\right\rangle$ of $n$th roots of unity then:

$$
[A(\beta)]^{n}=\left[A\left(\beta^{n}\right)\right]=[A(1)]=1 .
$$

Thus $m$ divides $n$. If $\sigma\left(\varepsilon_{n}\right)=\varepsilon_{n}^{t}$ then $\sigma\left(\varepsilon_{m}\right)=\varepsilon_{m}^{t}$. But then we have $\left[A\left(\beta^{o}\right)\right]=[A(\beta)]^{t}$. Hence $\left[A\left(\beta^{o}\right)\right]=[A(\beta)]$ if and only if $\sigma$ fixes $\varepsilon_{m}$. But $\left[A\left(X^{o}, K\right)\right]=\left[A\left(\beta^{o}\right)\right]$. Thus $\left[A\left(X^{o}, K\right)\right]=[A(X, K)]$ if and only if $\sigma$ fixes $\varepsilon_{m}$.

Maintaining the above notation we get:

Corollary 3.6. If $\sigma \in \operatorname{Aut}(K)$ then $[A(X, K)]=\left[A\left(X^{\sigma}, K\right)\right]$ if and only if $\sigma$ extends to Aut $(A)$.

Proof. In Mollin [13] we proved that $\sigma$ extends to Aut $(A)$ if and only if $\sigma$ fixes $\varepsilon_{m}$.

4. Uniform distribution and $S(K)$. We let $K / L$ be a finite Galois extension of number fields. A central simple algebra $A$ over $K$ is said to have uniformly distributed Hasse invariants relative to $L$ if the following are satisfied:

(4.1) If the index of $A$ is $m$ then $\varepsilon_{m}$ is in $K$, and:

(4.2) If $\mathscr{P}$ is a $K$-prime above the $L$-prime $\mathscr{P}$ and $\tau \in G(K / L)$ with $\varepsilon_{m}^{o}=\varepsilon_{m}^{b}$ then the $\mathscr{P}$-invariant of $A$ satisfies:

$$
\operatorname{inv}_{\mathscr{O}}(A) \equiv b \operatorname{inv}_{\mathscr{S}^{\sigma}}(A)(\bmod 1) .
$$

Now let $\mathscr{P}$ and $\mathscr{Q}$ be $K$-primes above some $L$-prime $\mathscr{P}$ and let $K_{\mathscr{S}}$ denote the completion of $K$ at $\mathscr{P}$. For an algebra $A$ with uniformly distributed invariants we have that $A \otimes K_{\mathscr{P}}$ and $A \otimes K_{\mathscr{C}}$ have the same index (see Mollin [12]). We denote the common value of the indices of $A \otimes K_{\mathscr{S}}$ for all $K$-primes $\mathscr{P}$ above $\mathscr{P}$ by $\operatorname{ind}_{\mathscr{P}} A$, called the $\mathscr{P}$-local index of $A$. The uniform distribution group for $K$ relative to $L$ is the subgroup of $B(K)$ consisting of classes having an algebra with uniformly distributed invariants relative to $L$. If $L=Q$ we let $U_{Q}(K)=U(K)$ and refer to this group as the absolute uniform distribution group for $K$.

The above is a generalization of Benard and Schacher [1, Th. 1 , p. 280], (see also [16, Th. 6.1, p. 89]). In fact $S(K)$ is a subgroup of $U_{L}(K)$, (see Mollin [12]). In Mollin [8-13] the relationship between $S(K)$ and $U(K)$ for $K / Q$ finite abelian is examined.

For a rational prime $p$ let $S(K)_{p}$ denote the $p$-primary part of $S(K)$. We let $K / Q$ be finite abelian and $Q\left(\varepsilon_{p^{a}}\right) \subseteq K \subseteq Q\left(\varepsilon_{n}\right)$ where $p^{a}$ is the highest power of $p$ dividing $n$. G. Janusz [7, Cor. 1, p. 350] shows that if $p$ does not divide $\left|Q\left(\varepsilon_{n}\right): K\right|$ then: 


$$
S(K)_{p}=K \otimes S\left(Q\left(\varepsilon_{p^{a}}\right)\right)_{p}
$$

where the tensor product is taken over $Q\left(\varepsilon_{p a}\right)$.

In Mollin [8] it is shown that if $p$ does not divide $\left|K: Q\left(\varepsilon_{p^{a}}\right)\right|$ then:

$$
U(K)_{p}=S(K)_{p}=K \otimes S\left(Q\left(\varepsilon_{p} a\right)\right)_{p} .
$$

That is, every element in $U(K)_{p}$ is in fact an element of $S(K)_{p}$ and is of the form $[K \otimes A]$ where $[A] \in S\left(Q\left(\varepsilon_{p^{a}}\right)\right)_{p}$.

C. Ford and G. Janusz [5] give, for each prime $p$, examples of fields $K$ for which (4.3) does not hold. Following Ford and Janusz we present, for each prime $p$, fields $K$ for which the second equality of (4.4) does not hold but for which the first equality does hold.

We let $m=p \cdot s$ where $p$ and $s$ are odd rational primes with $s \equiv 1(\bmod p)$ and $L=Q\left(\varepsilon_{m}\right)$ with $\langle\tau\rangle=G\left(L / Q\left(\varepsilon_{p}\right)\right)$. We let $K$ be the fixed field of $\langle\sigma\rangle$ where $\sigma=\tau^{(s-1) / p}$.

Theorem 4.5. (1) If $s \equiv 1\left(\bmod p^{2}\right)$ then $A$, where $[A] \in S(K)_{p}$, is not similar to $K \otimes B$ for all $[B] \in S\left(Q\left(\varepsilon_{p}\right)\right)_{p}=U\left(Q\left(\varepsilon_{p}\right)\right)_{p}$. In particular:

$$
S(K)_{p} \neq K \otimes S\left(Q\left(\varepsilon_{p}\right)\right)_{p} .
$$

Moreover: $U(K)_{p}=S(K)_{p}$.

(2) If $s \not \equiv 1\left(\bmod p^{2}\right)$ then

$$
U(K)_{p}=S(K)_{p}=K \otimes S\left(Q\left(\varepsilon_{p}\right)\right)_{p} .
$$

Proof. (1) First we show that $U(K)_{p}=S(K)_{p}$. By Mollin, [8], if $[A] \in U(K)_{p}$ with $\operatorname{ind}_{q}(A)>1$ for some prime $q$ then $q \equiv 1$ $(\bmod p)$ and conversely, given a prime $q \equiv 1(\bmod p)$ there exists $[A] \in U(K)_{p}$ with $\operatorname{ind}_{q}(A)=p$ and $\operatorname{ind}_{t}(A)=1$ for all primes $t \neq q$. We note that the latter does not necessarily hold for $p=2$. Now, that $[A]$ is in fact in $S(K)_{p}$ follows from Janusz [4, Th. 3, p. 267], and since the list of notation needed to state the aforementioned theorem is longer than the proof we do not reproduce it here, but rather leave the reader to verify the technical details.

Thus we have $U(K)_{p}=S(K)_{p}$. Now we show $S(K)_{p} \neq K \otimes S\left(Q\left(\varepsilon_{p}\right)\right)_{p}$. Assume $S(K)_{p}=K \otimes S\left(Q\left(\varepsilon_{p}\right)\right)_{p}$. Since $[A] \in S(K)_{p}$ then $[A]=$ $[K \otimes B]$ where $[B] \in S\left(Q\left(\varepsilon_{p}\right)\right)_{p}$. Now let $Q$ be a $K$-prime above the $Q\left(\varepsilon_{p}\right)$-prime $\gamma$ which lies over $q$. Then:

$$
\operatorname{inv}_{\mathscr{C}}(A) \equiv\left|K_{\mathscr{Q}}: Q_{q}\left(\varepsilon_{p}\right)\right| \operatorname{inv}_{\mathscr{C}} B(\bmod 1)
$$

(see Deuring [3]).

However, $p|| K_{\complement}: Q_{q}\left(\varepsilon_{p}\right) \mid$ and $\operatorname{ind}_{\mathscr{C}} B \leqq p$ (see Mollin [7]). Thus 
$\operatorname{inv}_{\mathscr{C}} A=0$, a contradiction. Hence

$$
S(K)_{p} \neq K \otimes S\left(Q\left(\varepsilon_{p}\right)\right)_{p} .
$$

(2) $q \equiv 1\left(\bmod p^{2}\right)$ then $p$ does not divide $\left|K: Q\left(\varepsilon_{p}\right)\right|$ then by Mollin [6] $U(K)_{p}=S(K)_{p}=S\left(Q\left(\varepsilon_{p}\right)\right)_{p} \otimes K$.

To find examples for the case $p=2$ we invoke Yamada [16, Th. 7.8, p. 107] from which it follows that if $K=Q(\sqrt{d})$ is a real quadratic field with $d$ not divisible by a prime congruent to 3 modulo 4 then $U(K)=S(K) \neq S(Q) \otimes K$. We have $U(K)=U(K)_{2}$ and $S(K)=S(K)_{2}$ by (4.1). We now have fields $K$ such that $U(K)_{2}=$ $S(K)_{2} \neq S(Q) \otimes K$. If $d$ is divisible by a prime congruent to 3 modulo 4 then $U(K)_{2} \neq S(K)_{2}=S(Q) \otimes K$, thus completing our task.

\section{The decomposition theorem.}

Definition 5.1. Let $S(K, q)_{p}$, (respectively $U(K, q)_{p}$ ) denote the subgroup of $S(K)_{p}$ consisting of all elements having $t$-local index 1 for $t \neq q$.

Janusz [6, p. 254] notes that one would like (for neatness sake) to assert that $S(K)_{p}$ is the direct sum of groups $S(K, q)_{p}$ as $q$ ranges over all primes. This, however, he proves is not in general true. We prove that for $p$ odd we get:

THEOREM 5.2. $U(K)_{p}$ is the direct sum of groups $U(K, q)_{p}$ as $q$ ranges over all primes.

Proof. By Mollin [8] there exists $[A(q)] \in U(K)_{p}$ with $\operatorname{ind}_{q} A(q)=$ $p^{a}$ and $\operatorname{ind}_{t} A(q)=1$ for all primes $t \neq q$ where $p^{a}$ is the highest $p$-power root of unity in $K$. Thus, it follows that any $[A] \in U(K)_{p}$ has the form $A=\pi A(q)^{c_{q}}$.

\section{REFERENCES}

1. M. Benard and M. Schacher, The Schur subgroup II, J. Algebra, 22 (1972), 378-385.

2. M. Deuring, Algebren, second edition, Springer Verlag, Berlin, 1968.

3. L. Dornhoff, Group Representation Theory, Part a., Marcel Dekker Inc., New York, 1971.

4. B. Fein and B. Gordon, Fields generated by characters of finite groups, J. London Math. Soc., (2), 4 (1972), 735-740.

5. C. Ford and G. Janusz, Examples in the theory of the Schur group, Bull. Amer. Math. Soc., 79 (1973), 1233-1235.

6. G. Janusz, The Schur group of an algebraic number field, Annals of Math., 103 (1976), 253-281.

7. — The Schur group of cyclotomic fields, J. Number Theory, 7 (1975), 345-352.

8. R. Mollin, Algebras with uniformly distributed invariants, J. Algebra, 44 (1977), 271-282.

9. Uniform distribution and the Schur subgroup, J. Algebra, 42 (1976), 
261-277.

10. R. Mollin, Uniform distribution and real fields, J. Algebra, 43 (1976), 155-167.

11. - $U(K)$ for a quadratic field $K$, Communications in Algebra, 4 (8), (1976), 747-759.

12. - Generalized uniform distribution of Hasse invariants, Communications in Algebra, 5 (3), (1977), 245-266.

13. - Herstein's conjecture, automorphisms, and the Schur group, Communications in Algebra, 6 (3), (1978), 237-248.

14. I. Reiner, Maximal Orders, Academic Press, New York, 1975.

15. P. Ribenboim, Algebraic Numbers, Wiley-Interscience, New York, 1972.

16. T. Yamada, The Schur Subgroup of the Brauer Group, Lecture Notes in Mathematics, No. 397, Springer-Verlag, 1974.

17. H. J. Zassenhaus, The Theory of Groups, 2nd Ed., Chelsea, New York, 1958.

Received July 15, 1977.

UnIVERSITY OF TORONTO

Toronto, Ontario, M5S 1A1 


\section{PACIFIC JOURNAL OF MATHEMATICS}

\section{EDITORS}

RICHARD ARENS (Managing Editor)

University of California

Los Angeles, CA 90024

Charles W. Curtis

University of Oregon

Eugene, OR 97403

C. C. MOORE

University of California

Berkeley, CA 94720

\section{J. DugundJI}

Department of Mathematics

University of Southern California

Los Angeles, CA 90007

R. FinN and J. Milgram

Stanford University

Stanford, CA 94305

\section{ASSOCIATE EDITORS}
E. F. BECKENBACH
B. H. NeumanN
F. WOLF
K. YosHIDA

\section{SUPPORTING INSTITUTIONS}

\author{
UNIVERSITY OF BRITISH COLUMBIA \\ CALIFORNIA INSTITUTE OF TECHNOLOGY \\ UNIVERSITY OF CALIFORNIA \\ MONTANA STATE UNIVERSITY \\ UNIVERSITY OF NEVADA, RENO \\ NEW MEXICO STATE UNIVERSITY \\ OREGON STATE UNIVERSITY \\ UNIVERSITY OF OREGON
}

\author{
UNIVERSITY OF SOUTHERN CALIFORNIA \\ STANFORD UNIVERSITY \\ UNIVERSITY OF HAWAII \\ UNIVERSITY OF TOKYO \\ UNIVERSITY OF UTAH \\ WASHINGTON STATE UNIVERSITY \\ UNIVERSITY OF WASHINGTON
}

The Supporting Institutions listed above contribute to the cost of publication of this Journal, but they are not owners or publishers and have no responsibility for its content or policies.

Mathematical papers intended for publication in the Pacific Journal of Mathematics should be in typed form or offset-reproduced, (not dittoed), double spaced with large margins. Please do not use built up fractions in the text of the manuscript. However, you may use them in the displayed equations. Underline Greek letters in red, German in green, and script in blue. The first paragraph or two must be capable of being used separately as a synopsis of the entire paper. Items of the bibliography should not be cited there unless absolutely necessary, in which case they must be identified by author and journal, rather than by item number. Manuscripts, in triplicate, may be sent to any one of the editors. Please classify according to the scheme of Math. Reviews, Index to Vol. 39. All other communications should be addressed to the managing editor, or Elaine Barth, University of California, Los Angeles, California, 90024.

50 reprints to each author are provided free for each article, only if page charges have been substantially paid. Additional copies may be obtained at cost in multiples of 50 .

The Pacific Journal of Mathematics is issued monthly as of January 1966. Regular subscription rate: $\$ 72.00$ a year (6 Vols., 12 issues). Special rate: $\$ 36.00$ a year to individual members of supporting institutions.

Subscriptions, orders for numbers issued in the last three calendar years, and changes of address should be sent to Pacific Journal of Mathematics, 103 Highland Boulevard, Berkeley, California, 94708. Older back numbers obtainable from Kraus Periodicals Co., Route 100, Millwood, NY 10546.

PUBLISHED BY PACIFIC JOURNAL OF MATHEMATICS, A NON-PROFIT CORPORATION

Printed at Kokusai Bunken Insatsusha (International Academic Printing Co., Ltd.). 8-8, 3-chome, Takadanobaba, Shinjuku-ku, Tokyo 160, Japan.

Copyright (C) 1978 by Pacific Journal of Mathematics Manufactured and first issued in Japan 


\section{Pacific Journal of Mathematics}

\section{Vol. 76, No. $2 \quad$ December, 1978}

Stephanie Brewster Brewer Taylor Alexander, Local and global convexity in complete Riemannian manifolds ...........................

Claudi Alsina i Català, On countable products and algebraic convexifications of probabilistic metric spaces ...............................

Joel David Berman and George Grätzer, Uniform representations of

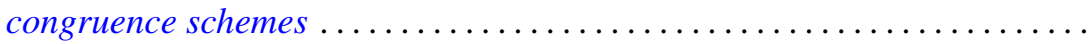

Ajit Kaur Chilana and Kenneth Allen Ross, Spectral synthesis in

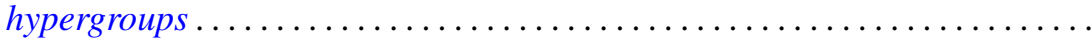

David Mordecai Cohen and Howard Leonard Resnikoff, Hermitian quadratic forms and Hermitian modular forms . .........................

Frank Rimi DeMeyer, Metabelian groups with an irreducible projective

representation of large degree .............................

Robert Ellis, The Furstenberg structure theorem .....................

Heinz W. Engl, Random fixed point theorems for multivalued mappings .......

William Andrew Ettling, On arc length sharpenings ..................

Kent Ralph Fuller and Joel K. Haack, Rings with quivers that are trees........

Kenneth R. Goodearl, Centers of regular self-injective rings ...............

John Gregory, Numerical algorithms for oscillation vectors of second order

differential equations including the Euler-Lagrange equation for

symmetric tridiagonal matrices.

Branko Grünbaum and Geoffrey Shephard, Isotoxal tilings

Myron Stanley Henry and Kenneth Leroy Wiggins, Applications of

approximation theory to differential equations with deviating

arguments

Mark Jungerman, The non-orientable genus of the n-cube .

Robert Richard Kallman, Only trivial Borel measures on $S_{\infty}$ are

quasi-invariant under automorphisms ................

Joyce Longman and Michael Rich, Scalar dependent algebras in the

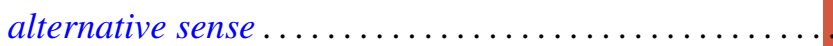

Richard A. Mollin, The Schur group of a field of characteristic zero ........ 471

David Pokrass, Some radical properties of rings with $(a, b, c)=(c, a, b) \ldots 479$

Margaret Shay and Paul Ruel Young, Characterizing the orders changed by

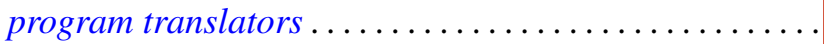

Jerrold Norman Siegel, On the structure of $B_{\infty}(F), F$ a stable space...

Surjeet Singh, (hnp)-rings over which every module admits a basic

submodule...

A. K. Snyder, Universal interpolating sets and the Nevanlinna-Pick property in

Banach spaces of functions...

Jeffrey D. Vaaler, On the metric theory of Diophantine approximation ... 